\title{
Subwavelength Microstructures Fabrication by Self-Organization Processes in Photopolymerizable Nanocomposite
}

\author{
I. Yu. Denisyuk, N. O. Sobeshuk, J. A. Burunkova, and N. D. Vorzobova \\ Saint-Petersburg National Research University of Information Technologies, Mechanics and Optics, Street Kronverskii 49, \\ Saint-Petersburg 197101, Russia \\ Correspondence should be addressed to I. Yu. Denisyuk, denisiuk@mail.ifmo.ru
}

Received 11 October 2011; Revised 11 December 2011; Accepted 2 January 2012

Academic Editor: Jaetae Seo

Copyright ( 92012 I. Yu. Denisyuk et al. This is an open access article distributed under the Creative Commons Attribution License, which permits unrestricted use, distribution, and reproduction in any medium, provided the original work is properly cited.

This paper describes our research results on nanometers sizes subwavelength nanostructure fabrication by UV curing of special nanocomposite material with self-organization and light self-focusing effects. For this purpose, special UV curable nanocomposite material with a set of effects was developing: light self-focusing in the photopolymer with positive refractive index change, self-organization based on photo-induced nanoparticles transportation, and oxygen-based polymerization threshold. Both holographic and projection lithography writing methods application for microstructure making shows geometrical optical laws perturbation as result of nanocomposite self-organization effects with formation of nanometers-sized high-aspect-ratio structures. Obtained results will be useful for diffraction limit overcoming in projection lithography as well as for deep lithography technique.

\section{Introduction}

The main problem of current photolithography is diminishing of minimal feature sizes up to subwavelength value. The smallest feature size $X_{\min }$ that can be projected by a coherent imaging system is $X_{\min }=\lambda / 2 \mathrm{NA}$, and the depth of focus DOF is DOF $=\lambda /\left[2 \mathrm{NA}^{2}\right]$, where $\lambda$ is the wavelength of the illumination, and NA is the numerical aperture. The most ordinary way to attain smaller feature sizes is to reduce the wavelength up to excimer laser wavelengths (248 or $193 \mathrm{~nm}$ ). The NA is typically between 0,5 and 0,8 , so the feature size is on the order of the exposure wavelength. State-of-the-art semiconductor fabrication facilities in the year 2010 are forecasted to use a $32 \mathrm{~nm}$ process, at which point the resolution and depth of focus constraints of optical lithography become difficult.

Figure 1 plots the resolution against the film stack's minimum refractive index. Note that the calculation includes 0.90 as the practical optics limit for $\sin (\theta)$. This requires that the refractive index of the immersion fluids be at least $10 \%$ higher than the theoretical minimum value. It is also assumed that all other refractive indices in the stack are higher than the fluid's refractive index. To overcome pitch size of $32 \mathrm{~nm}$, it is needed to use fluid with refractive index higher than 2,0 that is impossible.

Therefore, techniques to enhance the performance of optical lithography are very important now. As the fundamental limits of optical lithography are approached, the nonlinear optical properties of the photoresist become increasingly important. This nonlinearity arises from the change in the complex index of refraction of the photoresist under UV exposure. A common nonlinear optical phenomenon is the light-induced change in the imaginary part of the index of refraction $\sim$, that is, absorption upon exposure. For instance, contrast enhancement layers composed of a photobleachable dye are often overcoated on a photoresist $[1,2]$ to introduce exposure thresholding.

For the same reason, special UV-curable nanocomposite with strong non-linear and self-writing effects overcoated on photoresist to improve light distribution in the spot can be used.

The same proposed technique is applicable for deep lithography based on $365 \mathrm{~nm}$ UV light with high scattering to improve the shape of small feature in results of geometrical optical laws perturbation at use of light self-focusing in materials. 


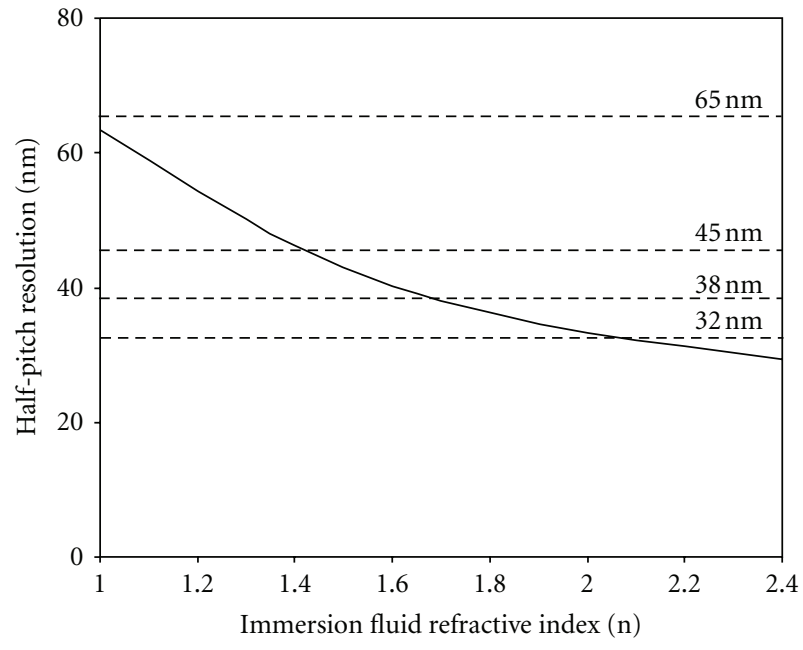

FIGURE 1: Half-pitch resolution plotted against immersion fluid refractive index according to the work [3].

If we make nanocomposite system with self-writing effects and place it as a topcoat, we will obtain self-writing subwavelength artificial waveguide that will guide the light to small subwavelength spot on photoresist surface.

So to make it, it is necessary to develop special material with self-writing effects.

\section{Experimental Conditions}

UV-curable composition has been developed previously and was described in our works $[5,6]$. Acrylic monomers were used such as composition of 2-carboxyethyl acrylate, bisphenol A glycerolate diacrylate, 1,6-gexanediol diacrylate, and trimethylolpropane ethoxylate $(1 \mathrm{EO} / \mathrm{OH})$ methyl ether diacrylate (in ratio 5:2:1:2 accordingly) from Aldrichliquid composition and 2-carboxyethyl acrylate, RSX 51027 (from USB) (in ratio 1:3 accordingly)—solid composition, as initiator was used 2,2-dimethoxy-2-phenylacetophenone (from Aldrich). Initiator type and concentration $(0,2 \mathrm{wt} \%)$ were chosen according to light source $\lambda=320--365 \mathrm{~nm}$. Concentration of initiator allows light penetration in monomer material on $1 \mathrm{~mm}$.

For projection lithography were used two monomer compositions: 2-carboxyethyl acrylate and bisphenol A glycerolate in ratio $70: 30$ and bisphenol A glycerolate diacrylate, 2-carboxyethyl acrylate, and phenoxyethyl acrylate in ratio $25: 55: 20$ with nanoparticles.

Nanocomposites used in this work were based on $\mathrm{ZnO}$ nanoparticles dispersed in monomers. The concentration of nanocrystals in the composite which is about $10 \mathrm{wt} \%$ and their size $(20 \mathrm{~nm})$ will ensure low light scattering by the nanocomposite. $\mathrm{ZnO}$ nanocrystals were purchased from Russian local supplier. Prepared nanoparticles were mixed with UV curable monomers mixture. Nanocomposite preparation method and its properties were described previously [6-8].
All experiments were accomplished at room temperature in air without special inert atmosphere (argon). To reduce inhibition effect of oxygen, nanocomposite was cured between two substrates-glass and polyester film.

Process of microstructural element formation was made as follows: drop of composition was placed on substrate (in our case glass processed by $\mathrm{NaOH}$ ) having chemical interaction with acid groups of composition and covered by polyester film with thin layer of release coating (siloxane). Thickness of composition layer was determined by spacers. UV curing took place at UV irradiation of composition by use of holographic technique or by projection lithography. After curing polyester was removed, and substrate was washed by isopropyl alcohol to remove noncured composition. Holographic method based on interferometer with $320 \mathrm{~nm}$ He-Cd CW laser source. In current experiment angle between two laser beams was 10 degrees, and writing lattice period was $-2 \mu \mathrm{m}$.

Projection lithography was carried out based on DPSS laser source $(355 \mathrm{~nm})$ and quartz lens with aperture 0,4 .

SEM photos as well as ion etching of the materials were made by ion beam tool-CrossBeam Neon 40 EsB (Carl Zeiss).

\section{Results and Discussion}

Light self-focusing and self-organization effects at UV curing of acrylate-based nanocomposites were investigated previously [9]. There are a few effects as follows.

3.1. Self-Focusing of the Light in Material with Positive Refractive Index Change at Photopolymerization. Effect of light selffocusing in optical material having proprieties of positive change of refractive index (RI) at light action is widely investigated recently. For example, in cited work, the results of light self-focusing and self-written waveguide preparation process obtained on glass light-sensitive material are summarized [10].

Self-written waveguides can be evolved if a Gaussian laser beam is focused onto and allowed to propagate through a photosensitive material that shows positive refractive index change in response to laser exposure. Since the refractive index increases with intensity, the initially diffracting beam causes the refractive index to build up along the propagation axis, which is reflected by narrowing and increasing peak intensity of the outcoming beam. In the early stages, an adiabatic taper forms, and over time, a channel waveguide can be created throughout the sample. These are called self-written waveguides as the same light that induced the waveguide is also guided by it [10]. Our experiments show important influence of the well-known oxygen inhibition action on reinforcement of self-focusing light in photopolymer. Oxygen inhibition action to acrylate photopolymerization is described previously [11].

3.2. Short-Distance Nanoparticles Transportation. Effect of light-induced nanoparticles redistribution in nanocomposite is a new effect discovered recently. It takes place at 


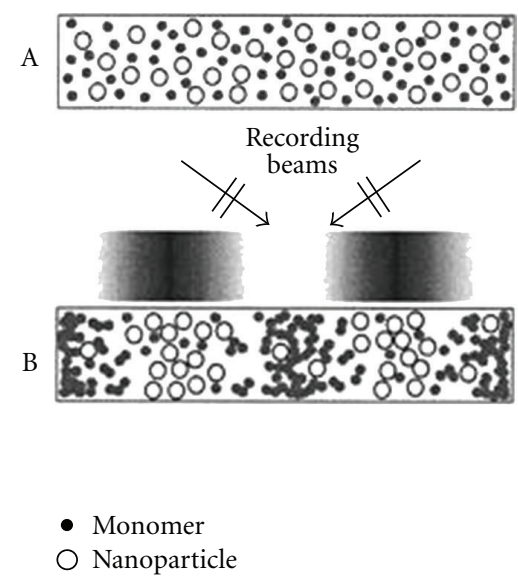

(a)

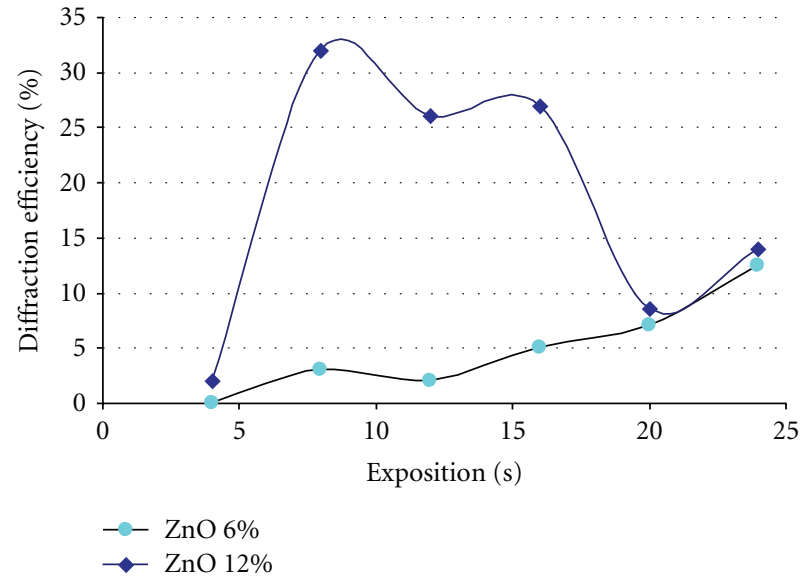

(b)

FIGURE 2: (a) Nanoparticles transportation in photopolymer according to the work in [4], (b) our experimental diffraction efficiency dependence on exposition and nanoparticles concentration.

photopolymeric nanocomposite irradiation by periodic light distribution, for example, by lattice made by interference of two laser beams.

The first time these processes were found by Suzuki and coworkers in 2005 on organic-inorganic nanocomposite photopolymer system in which inorganic nanoparticles with a larger refractive index differ from photopolymerized monomers are dispersed in uncured monomers [12]. Inorganic materials possess a wide variety of refractive indices that give us the opportunity to obtain much higher refractive index changes $\Delta n$ than conventional photopolymers with low scattering loss [13].

Explanation of effects was made in the work of [4]. For monomers with radical photopolymerization, spatially nonuniform light illumination will produce free radicals by dissociation of initiators and subsequent reaction of free radicals with monomers, which leads to chain polymerization of individual monomers in the bright regions.

This polymerization process lowers the chemical potential of monomers in the bright regions, leading to diffusion (short-distance transportation) of monomers from the dark to the bright regions. On the other hand, photoinsensitive inorganic nanoparticles have diffusion from the bright to the dark regions, as illustrated in Figure 2(a), since the particles are not consumed, and their chemical potential increases in the bright regions as a result of the monomer consumption. Such a mutual diffusion process continues until the monomers are consumed completely by polymerization processes and until the high viscosity of cured material makes monomers and nanoparticles immobile. As a result, the spatial distribution of nanoparticles is also fixed, and a refractive-index grating is created as a result of compositional and density differences between the bright and the dark regions.

Figure 2 shows diffraction efficiency dependence of photopolymerizable nanocomposite material from exposition time and nanoparticles concentration. Unlike classic holographic photopolymer in our material, unpolymerized

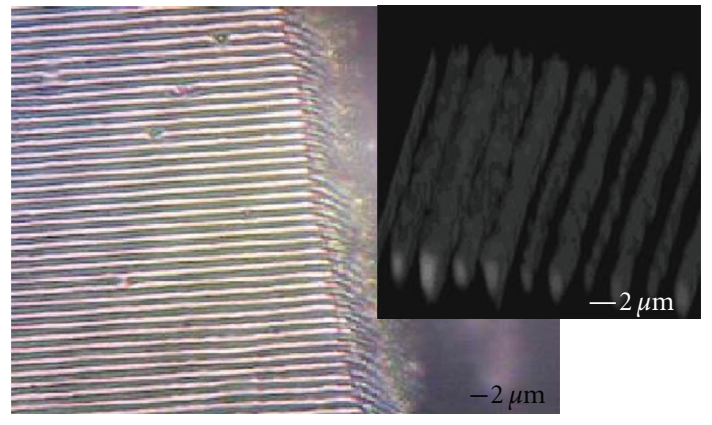

FIGURE 3: Microphotos of cut of obtained 3D structure (leftoptical and right—confocal microscopy), lattice period- $2 \mu \mathrm{m}$.

materials were dissolved by alcohol and removed after exposition. According to schema (Figure 2(a)), unpolymerized monomer in dark areas was removed by dissolution. High augmentation of diffraction efficiency at $12 \%$ nanoparticles concentration is a result of nanoparticles redistribution.

As a result, we will obtain $3 \mathrm{D}$ parallel plates of polymerized material with nanoparticles divided by the same plate without nanoparticles. Our measurement of the plate was made by transmission optical microscopy on micro-cut of structure and the same obtained by confocal microscopy shown in Figure 3. According to Figure 2(b) there takes place short-distance nanoparticles transportation to the bright regions with change of solubility of material and reinforcement of polymerized 3D lattice formation.

So the process of nanoparticles displacement is a selfwriting process or reinforcement of self-focusing process as discussed above.

After that the first experiments on nanoparticles redistribution in nanocomposite transformation on high aspect ratio nanosized structures were made. Figure 4 shows result of ion etching of previously made holographic micropatterns in nanocomposite. 


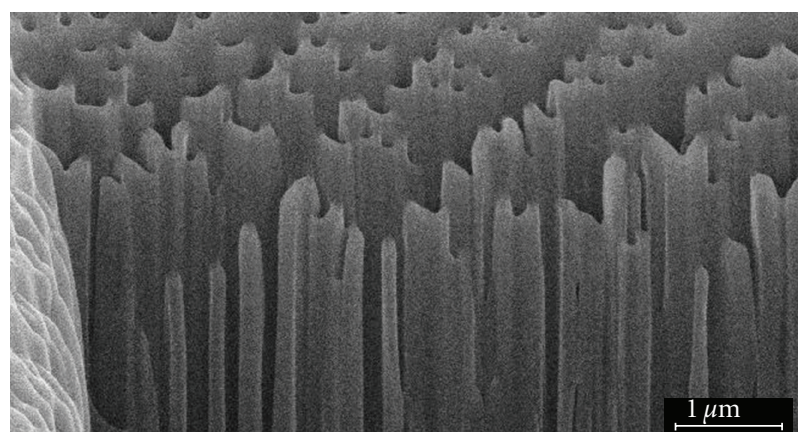

FIGURE 4: The result of ion beam etching: nanocomposite areas with formation of vertical $100 \mathrm{~nm}$ width elements.

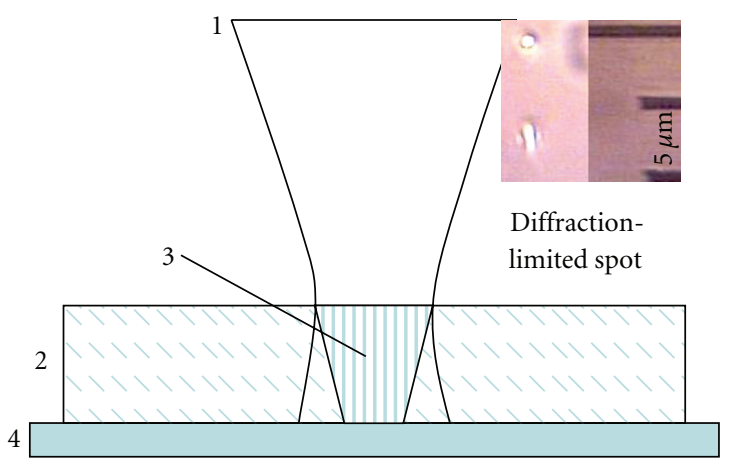

Figure 5: Model of light redistribution behind the lens spot as a result of self-writing processes. (1) UV light beam, (2) nanocomposite, (3) Tip self-writing, and (4) photoresist. Polymerized small one-micron-sized cylinder with vertical borders made by this process (insert).

We think that ion beam etches nanoparticles-free polymer plates and does not touch nanoparticles enriched plates. As a result, after polymer evaporation nanoparticles redistribution becomes visual, etching forms subwavelength nano-sized columns with high aspect ratio.

3.3. Microstructure Writing by Projection Lithography. Selfwriting and self-focusing effects discussed above are applicable for diminishing polymerized area initially corresponding to light distribution in objective spot as well as to overcome geometric distribution of the light in focus. The main effect is light self-focusing that can be reinforced by oxygen inhibition $[14,15]$ and nanoparticles redistribution at photopolymerization. Figure 5 shows proposed application of self-writing processes in projection photolithography: using nanocomposite with self-writing effects overcoated on photoresist to improve light distribution in the spot on the photoresist surface.

For comparison, two monomer compositions (with and without nanoparticles) were used, and results of them are different. In Figure 6 are represented polymer microstructures obtained in case of 2-carboxyethyl acrylate and bisphenol A glycerolate composition (without nanoparticles). Relation between dimensions/structure shape and exposition is observed. In this experiment was used projection of spot

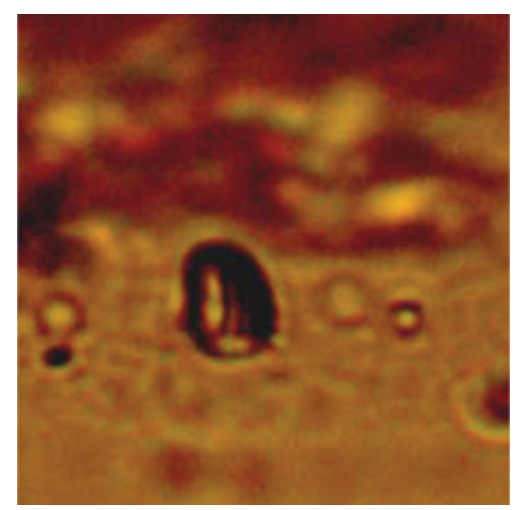

(a)

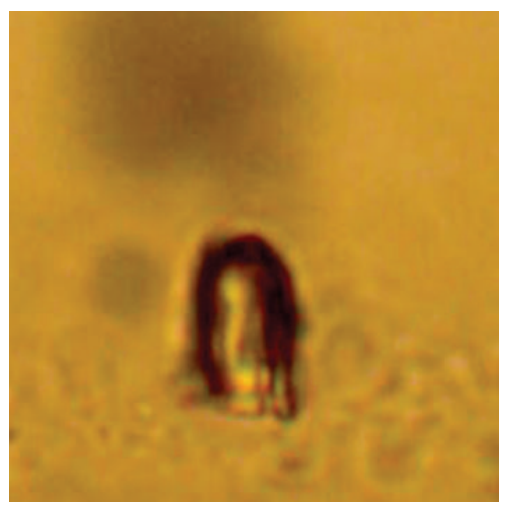

(b)

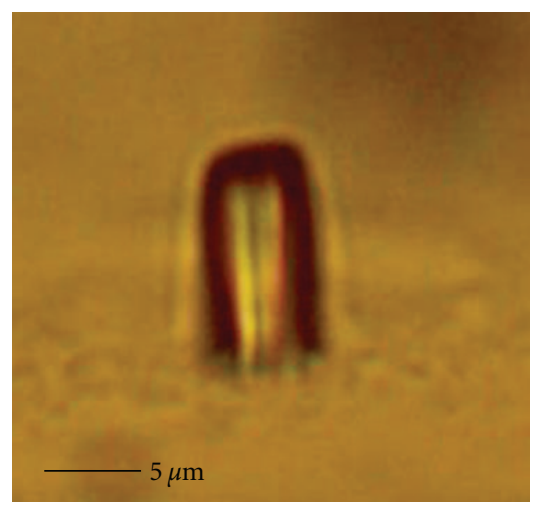

(c)

Figure 6: Structures obtained from photopolymer without nanoparticles: (a) diameter $3 \mu \mathrm{m}$ (spot size $2 \mu \mathrm{m}$ ); (b) diameter $4 \mu \mathrm{m}$ (spot size $4 \mu \mathrm{m}$ ); (c) diameter $6.5 \mu \mathrm{m}$ (spot size $6 \mu \mathrm{m}$ ).

expected diameter 1, 2, 4, and $6 \mathrm{~mm}$. Composition without nanoparticles does not allow to obtain structures with all expected diameters. All cylinders based on this composition were at least 3-4 $\mu \mathrm{m}$ diameter; in case of $1 \mu \mathrm{m}$ expected spot size formation of elements did not take place.

Elements form tends to the cone that corresponds to energy distribution in spot.

In contrast with this series, experiments with nanocomposite gave different results-we made structures with expected diameters $1 \mathrm{~mm}$ and obtained diameter less than $1 \mu \mathrm{m}$ with a form near to cylinder in Figure 7. 


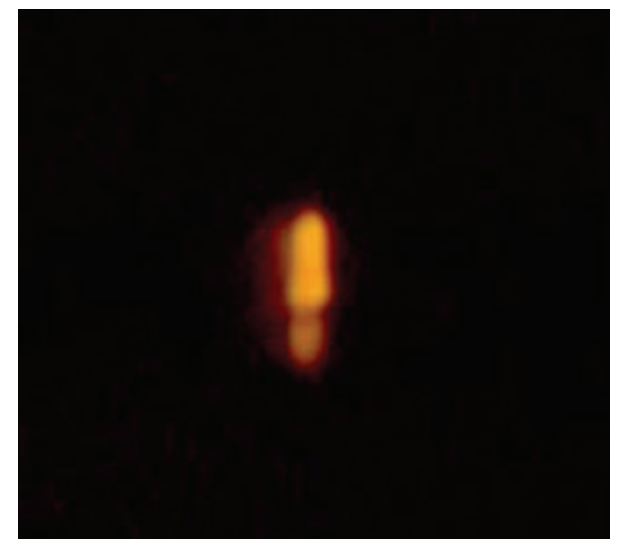

(a)

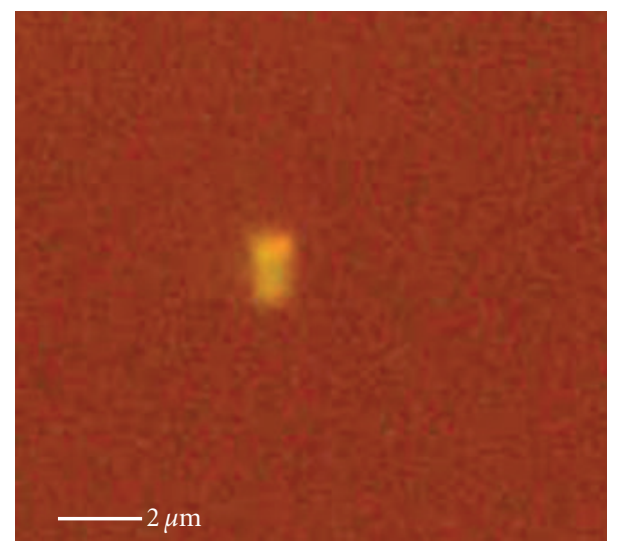

(b)

FIGURE 7: $2 \mu \mathrm{m}$ height structures obtained from nanocomposite: (a) diameter $0.7 \mu \mathrm{m}$ (spot size $1,5 \mu \mathrm{m}$ ), (b) diameter $0.6 \mu \mathrm{m}$ (spot size $1,5 \mu \mathrm{m})$.

Figure 7 shows that the use of nanocomposite gives formation of subwavelength elements formed with geometric optics law violation (formation of self-writing cylinder in dispute to cone).

In fact, used lens should form cone 20 degrees, but as a result of nanocomposite self-organization, it is the form of cylinders with vertical borders that confirms our guess-work on self-organization effects in photolithography.

\section{Conclusion}

Nanocomposite photopolymerizable material shows a set of self-writing effects due to the light beam redistribution as a result of self-focusing in non-linear photopolymer (positive refractive index change at photopolymerization) and effects of photopolymerization inhibition by oxygen and short-distance nanoparticles displacement. UV curing of nanocomposite will result in reinforcement of light selffocusing and grasping of polymerized area. The process is applicable for photolithography. The experiment made at use of 0,4 aperture lens shows possibility of transformation of initial conical light distribution to cylindrical one as a result of investigated processes in nanocomposite UV-curable material and formation of subwavelength-sized elements.

\section{Acknowledgment}

The work was made under support of Grant of Russian Ministry of Education no. RNP.2.1.1.3937, "Investigation of possible way for overcoming diffraction limit in photolithography by use of self-organization and non-linear proprieties of photopolymerizable nanocomposite materials," 20092011.

\section{References}

[1] B. F. Griffing and P. R. West, "Contrast enhanced lithography," Solid State Technology, vol. 28, no. 5, pp. 152-157, 1985.

[2] C. Rosilio, A. Rosilio, and B. Serre, "Charge-transfer complexes applied to photolithography by formation of a portable conformable mask and as a contrast-enhancement layer," Microelectronic Engineering, vol. 8, no. 1-2, pp. 37-54, 1988.

[3] H. Sewell, D. McCafferty, L. Markoya, E. Hendrickx, J. Hermans, and K. Ronse, "32nm node technology development Using interference immersion lithography," in Advances in Resist Technology and Processing XXII, vol. 5753 of Proceedings of SPIE, pp. 491-501, 2005.

[4] Y. Tomita, N. Suzuki, and K. Chikama, "Holographic manipulation of nanoparticle distribution morphology in nanoparticle-dispersed photopolymers," Optics Letters, vol. 30, no. 8, pp. 839-841, 2005.

[5] T. V. Smirnova, Y. E. Burunkova, and I. Y. Denisyuk, "Measuring the shrinkage of UV-hardenable composites based on acrylates and diacrylates," Journal of Optical Technology, vol. 73, no. 5, pp. 352-355, 2006.

[6] J. A. Burunkova, I. Yu. Denisyuk, N. N. Arefieva, and S. A. Semina, "Influence of $\mathrm{SiO}_{2}$ nanoaddition on the self-organization via UV-polymerization of acrylate nanocomposites," Molecular Crystals and Liquid Crystals, vol. 536, pp. 242-248, 2011.

[7] T. R. Williams, I. Yu. Denisyuk, and J. E. Burunkova, "Hybrid optical material based on high nanoparticles concentration in UV-curable polymers-technology and proprieties," Molecular Crystals \& Liquid Crystals, vol. 497, no. 1, pp. 142-153, 2008.

[8] J. E. Burunkova, I. Y. Denisyuk, and T. R. Williams, "Filled polymers with high nanoparticles concentration-synthesis and properties," Journal of Applied Polymer Science, vol. 116, no. 4, pp. 1857-1866, 2010.

[9] I. Yu. Denisyuk, N. D. Vorzobova, J. E. Burunkova, N. N. Arefieva, and M. I. Fokina, "Self-organization effects in photopolymerizable nanocomposite," Molecular Crystals and Liquid Crystals, vol. 536, no. 1, pp. 233-241, 2011.

[10] T. M. Monro, D. Moss, M. Bazylenko, C. Martijn De Sterke, and L. Poladian, "Observation of self-trapping of light in a self-written channel in a photosensitive glass," Physical Review Letters, vol. 80, no. 18, pp. 4072-4075, 1998.

[11] E. Andrzejewska, "Photopolymerization kinetics of multifunctional monomers," Progress in Polymer Science (Oxford), vol. 26, no. 4, pp. 605-665, 2001.

[12] N. Suzuki, Y. Tomita, and T. Kojima, "Holographic recording in $\mathrm{TiO}_{2}$ nanoparticle-dispersed methacrylate photopolymer films," Applied Physics Letters, vol. 81, no. 22, pp. 4121-4123, 2002.

[13] N. Suzuki, Y. Tomita, K. Ohmori, M. Hidaka, and K. Chikama, "Highly transparent $\mathrm{ZrO}_{2}$ nanoparticle-dispersed acrylate photopolymers for volume holographic recording," Optics Express, vol. 14, no. 26, pp. 12712-12719, 2006. 
[14] I. Y. Denisyuk, M. I. Fokina, N. D. Vorzobova, Y. E. Burunkova, and V. G. Bulgakova, "Microelements with high aspect ratio prepared by self-focusing of light at UV-curing," Molecular Crystals and Liquid Crystals, vol. 497, pp. 228-235, 2008.

[15] M. I. Fokina, N. O. Sobeshuk, and I. U. Denisyik, "Polymeric microelement on the top of the fiber formation and optical loss in this element analysis," Natural Science, vol. 2, no. 8, pp. 868-872, 2010. 

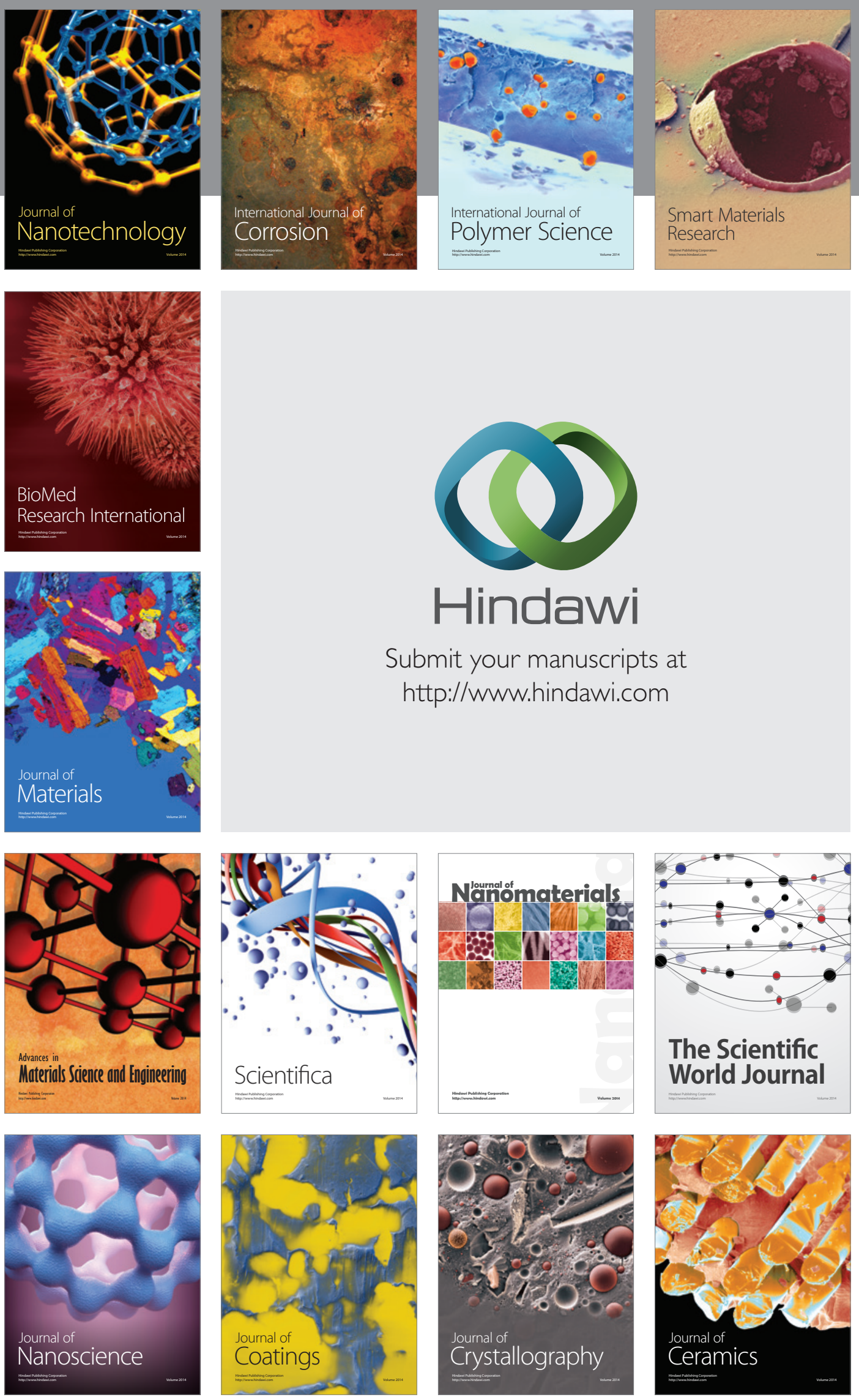

The Scientific World Journal

Submit your manuscripts at

http://www.hindawi.com

\section{World Journal}

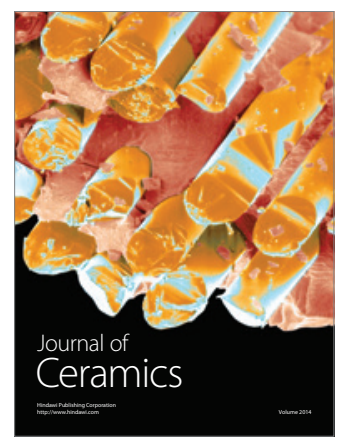

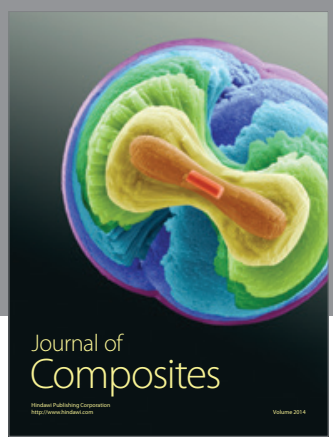
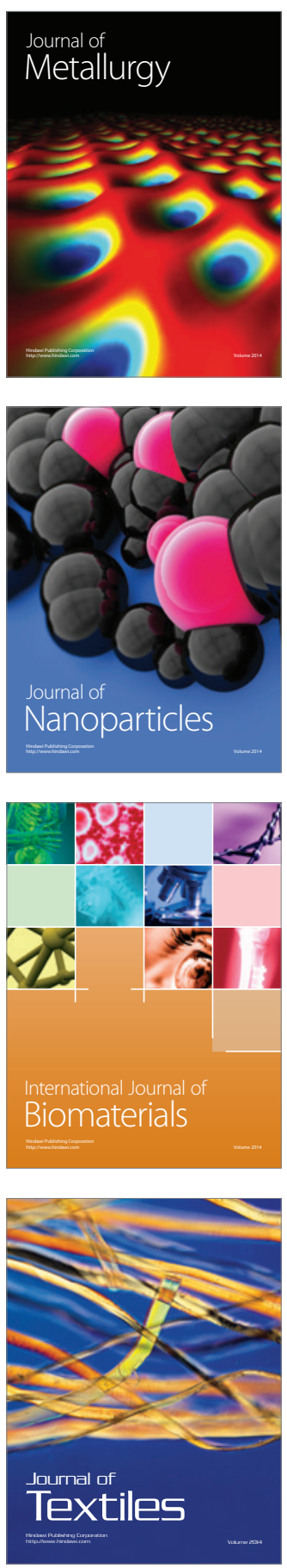